\title{
SPECIALIZED FITNESS PROGRAM WITH SWISS BALLS TO DEVELOP PHISICAL QUALITIES FOR STUDENTS
}

\author{
L. Lapadatov ${ }^{1}$, G. Dyakova ${ }^{1}$, N. Gocheva ${ }^{1}$, P. Stoycheva ${ }^{2}$, T. Andreev ${ }^{1}$ \\ ${ }^{1}$ Trakia University, Stara Zagora, Bulgaria, \\ ${ }^{2} 11$ elementary school "N. Liliev", Stara Zagora, Bulgaria
}

\begin{abstract}
An experiment has been carried out with 64 students in the first and second courses of all specialties of the Faculty of Economics, Faculty of Agriculture, and Faculty of Veterinary Medicine at the Thracian University of Stara Zagora. The purpose of this investigation isto improve the physical qualities of femalestudents through a specialized fitness program. Theoretical analysis, Pedagogical experiment, Sports-pedagogical testing, Pedagogical observation, Variation analysis, Graphical analysis and Comparative analysis have been applied to achieve the objective of the study. The results of the specialized swiss ball fitness program show an increase in physical qualities and anthropometric measurements. The results of our analysis indicate that the research is successful, the fitness program developed is effective and it gives us reason to believe that the inclusion of systemic Swiss ball exercises in the physical education and sport curriculum at the higher school will have a positive effect on physical qualities of the female students.
\end{abstract}

Key words: university, experiment

\section{INTRODUCTION}

The motor capabilities of human being reveal under the form of certain movements. Movements in physical culture assume more purposeful character uniting elaborate complexes of physical exercises. Motor action is distinguished by its own specific dynamic and kinematic characteristics. In the theory of physical education it is accepted for the separate sides of motor capabilities of human being to be defined as physical qualities (speed, strength, endurance, flexibility, skill) (1-5).

In the course of 40 years, the inflatable Swiss ball finds wide application in aerobic gymnastics, fitness, in various programmes for medical gymnastics, as well as with a number of other pathological conditions of motorsupport system. In contrast to the exercise devices, this ball does not give steady support, and almost every exercise with it engages great number of stabilizing muscles. The mere

\footnotetext{
*Correspondence to: Lyuben Lapadatov, Trakia University, Stara Zagora, Bulgaria, e-mail: luben82@mail.bg, GSM 0895992298
}

sitting on it places the body at unstable position, straining the muscles of legs and abdomen, in order to keep the balance. Apart from that, the elasticity of the pelvic bottom is improved, the breathing function is relieved, abdominal, spinal and pelvic muscles are strengthened. Endurance, strength, mobility, coordination and physical condition are increased significantly. For female students, the Swiss ball offers a new and different loading, uniting aerobic and force training at the same time. (6).

In its base, the circuit training is a serial (continuous or at intervals) repetition of several types of physical exercises, selected and united in a complex, in conformity to a certain orderly scheme. The purpose of the circuit training is to improve the aerobic capacity, and subsequently to increase the muscle strength and endurance. Circuit training achieves great energy consumption; improves the aerobic capacity; characterizes with low local fatigue; follows prompt restoration of forces and it is easy to adapt to any training plan. All these factors, and the fact that in the complex of the circuit training normally are 
included technically simple movements, welltrained in advance make it extremely suitable for educative and training purposes. $(7,8)$.

Anthropometric information of the body and limbs as a whole, and their separate parts or segments, gives a correct idea of the body harmonious development, of its proportion and of the conformity with the acting norms of healthy development. This imposes more detailed revealing of the dependencies between the body structure and functional capabilities of human being, perfection of the methods for their investigation, studying the mechanism of adaptation of organism to various types of sport activity and the dynamic changes occurred in the structure of the human body under the influence of the training loading. (914).

Purpose of investigation is the improvement of physical qualities of students through a specialized fitness programme.

\section{Tasks}

1. Investigation of literature sources.

2. Development of test battery.

3. Development and experimenting of a specialized fitness programme with Swiss balls.

4. Analyzing of results got.

\section{METHODOLOGY}

Specialized fitness programme with Swiss balls has been developed. The pedagogical experiment has been carried out in the course of 15 weeks within one educational semester.

64 first and second year students totally (female) have been investigated, from the Economic faculty, Veterinarian-Medical faculty and Agrarian faculty with Trakia University - Stara Zagora. The investigated persons have been divided into two groups experimental and control one. Experimental group consisted of 34 students, and the control one of 30 students. Investigated persons are of the age 19,6 years.

For achieving the aim of the investigation and for solving the tasks set, we have applied the following methods: Theoretic analysis, Sportpedagogical testing, Pedagogical experiment, Variation analysis, Comparative analysis, Graphic analysis.

\section{RESULTS AND ANALYSIS}

For investigation of the physical development and dynamics of the physical qualities we have applied a test battery, including 15 tests anthropometric indices are 7 and 8 are for measuring the physical qualities and morphofunctional indices (Table 1).

Table 1. Test battery

\begin{tabular}{|l|l|l|l|l|}
\hline \multicolumn{2}{|l|}{} & Measure unit & $\begin{array}{l}\text { Accuracy of } \\
\text { measurement }\end{array}$ & $\begin{array}{l}\text { Direction of } \\
\text { increase }\end{array}$ \\
\hline I. ANTHROPOMETRIC INDICES & $\mathrm{kg}$ & 1 & + \\
\hline 1 & Muscle mass & $\mathrm{cm}$ & 1 & + \\
\hline 2 & Chest measurement & $\mathrm{cm}$ & 1 & + \\
\hline 3 & Waist measurement & $\mathrm{cm}$ & 1 & + \\
\hline 4 & Measurement of right armpit & $\mathrm{cm}$ & 1 & + \\
\hline 5 & Measurement of left armpit & $\mathrm{cm}$ & 1 & + \\
\hline 6 & Measurement of right thigh & $\mathrm{cm}$ & 1 & + \\
\hline 7 & Measurement of left thigh & \multicolumn{2}{l|}{} \\
\hline $\begin{array}{l}\text { II. TESTS FOR MEASUREMENT OF PHYSICAL QUALITIES AND MORPHO-FUNCTIONAL } \\
\text { INDICES }\end{array}$ & $\mathrm{n}$ & 0.1 & \\
\hline 1 & Abdominal presses till refusal & 1 & 0.1 & + \\
\hline 2 & Vital capacity & $\mathrm{kg}$ & 1 & + \\
\hline 3 & Deadlift & $\mathrm{kg}$ & 1 & + \\
\hline 4 & Dynamometry suitable hand & $\mathrm{kg}$ & 1 & + \\
\hline 5 & Dynamometry unsuitable hand & $\mathrm{s}$ & 1 & + \\
\hline 6 & Plank & $\mathrm{s}$ & 1 & + \\
\hline 7 & Shuttle 10x10 & $\mathrm{cm}$ & 1 & - \\
\hline 8 & Vertical jump & \multicolumn{2}{|l|}{} \\
\hline
\end{tabular}

After analysing the incoming and out-coming anthropometric tests of the Control group we have noticed minimum increasing of the average test values: chest measurement, measurement of right and left armpit and measurement of left and right thigh. On the test "Muscle mass" we have noticed decreasing of the average valued of the Control group $(0,72$ 
$\mathrm{kg}$ ). With the Experimental group, we have noticed insignificant change in the average values of tests: muscle mass, chest measurement, dynamometry - suitable and unsuitable hand and measurement of left and right thigh.

There is a more significant change in the indices of the average values of the Control and Experimental group at the test „Waist measurement". At the Control group they have increased by $2,57 \mathrm{~cm}$, and at the Experimental one - no change has been noticed. Graphically, the results are shown on (Figure1).

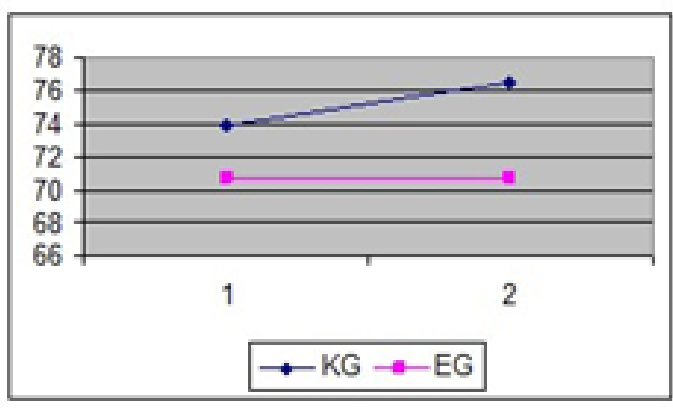

Figure 1. Waist measurement

At the „Abdominal presses till refusal” test, Control group has shown average values of the incoming tests 41,86 in number, and of the outcoming ones - 40,4 in number. Decreasing of the average values by 1,46 in number has been observed. Experimental group has shown average values of incoming tests 29,7 in number, and of the out-coming ones - 41,9. Improvement of the average values by 12,2 has been observed. After the application of the specialized fitness programme, the increasing of the average values from the "Abdominal presses till refusal" test of the Experimental group compared to the Control group is 13,66. Graphically, the results are shown on (Figure 2)

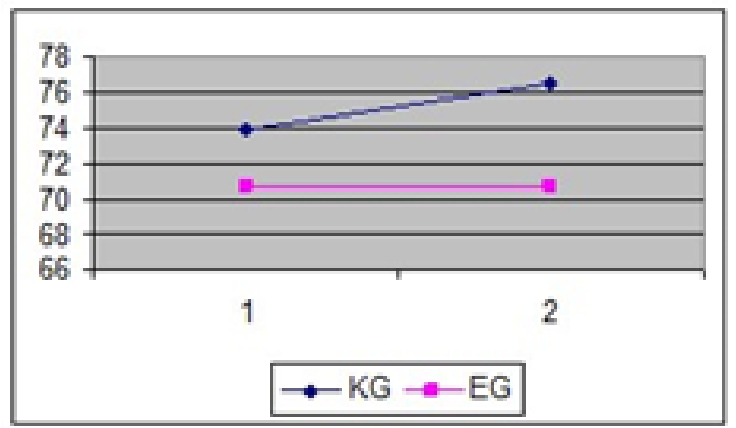

Figure 2. Abdominal presses till refusal

On the test „Vital capacity” the average values of the Control group of the incoming tests are 3,66 1 and of the out-coming ones - 3,62 1. No significant change of the average values is observed. Experimental group shows average values with the incoming measurement of 3,5 1 , and of the out-coming measurement - 4,2 1 . We have noticed an increase of 0,71 . As a result of the increasing of the average values of the test of the Experimental group, after the application of the specialized fitness programme with Swiss balls, increasing of the physical quality endurance of students is logically expected. Graphically, the results are shown on (Figure 3).

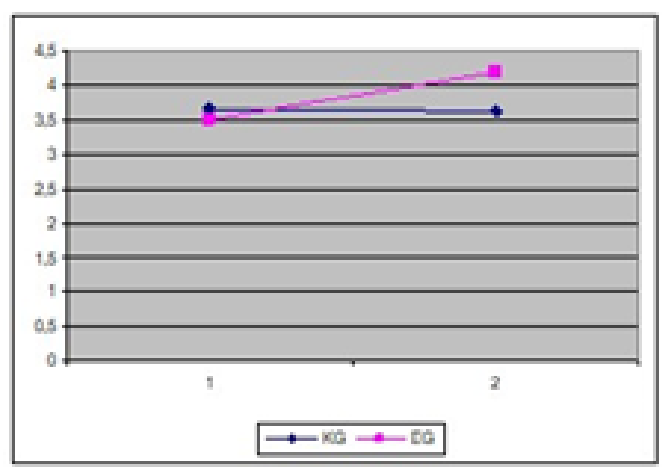

Figure 3. Vital capacity

On the „Deadlift” test at the first measurement, the Control group has shown average values of $53,3 \mathrm{~kg}$, and at the second measurement $52,16 \mathrm{~kg}$. There is decreasing of the average values by $1,14 \mathrm{~kg}$. The Experimental group on the first testing has shown average values of $63,3 \mathrm{~kg}$, and on the second measurement $-70,6$ $\mathrm{kg}$. Increasing of the results by $7,3 \mathrm{~kg}$ has been noticed. After the application of the specialized fitness programme, the increasing of the average values of the "Deadlift" test of the Experimental group compared to the Control group is $8,44 \mathrm{~kg}$. The extremely high results, reported with this test are due to the purposeful work with the new methods. Graphically, the results are shown on (Figure 4).

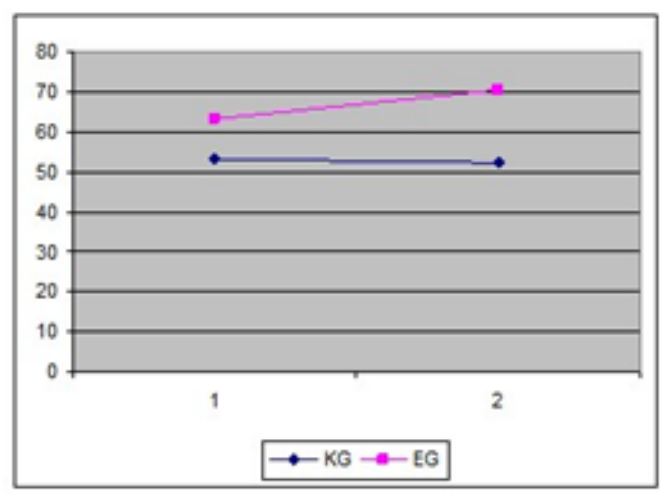

Figure 4. Deadlift

On the test „Dynamometry suitable hand”, the Control group has shown average values of incoming tests $14,3 \mathrm{~kg}$, and of the out-coming ones - 13,93 kg. Decreasing of the average values by $0,37 \mathrm{~kg}$ has been observed. The Experimental group has shown average values 
of the incoming tests of $11,5 \mathrm{~kg}$, and of the out-coming ones - 13,2 kg. Improvement of the average values by $1,7 \mathrm{~kg}$ has been observed. After the application of the specialized fitness programme, the increasing of the average values of the "Dynamometry suitable hand" test of the Experimental group compared to the Control group is $2,07 \mathrm{~kg}$. Graphically, the results are shown on (Figure 5).

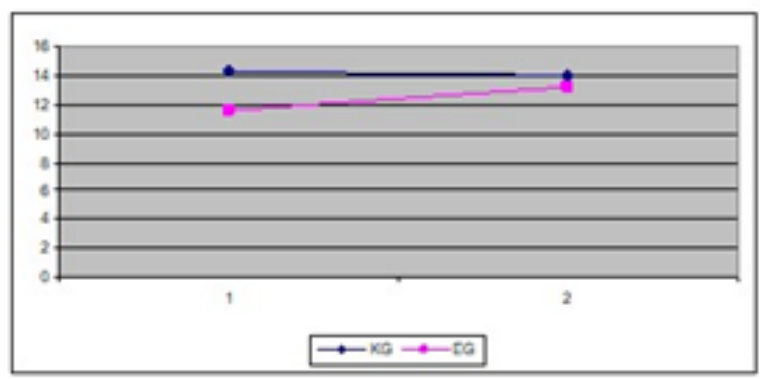

Figure 5. Dynamometry suitable hand

On the test „Dynamometry unsuitable hand”, the Control group has shown average values of incoming tests $13,26 \mathrm{~kg}$, and of out-coming $12,6 \mathrm{~kg}$. Decreasing of the average values by $0,66 \mathrm{~kg}$ has been observed. The Experimental group has shown average values of incoming tests of $10,1 \mathrm{~kg}$, and of the out-coming - 11,9 $\mathrm{kg}$. Improvement of the average values by 1,8 $\mathrm{kg}$ has been observed. After the application of the specialized fitness programme, the increasing of the average values of the "Dynamometry unsuitable hand" test of the Experimental group compared to the Control group is $1,46 \mathrm{~kg}$. Graphically, the results are shown on (Figure 6).

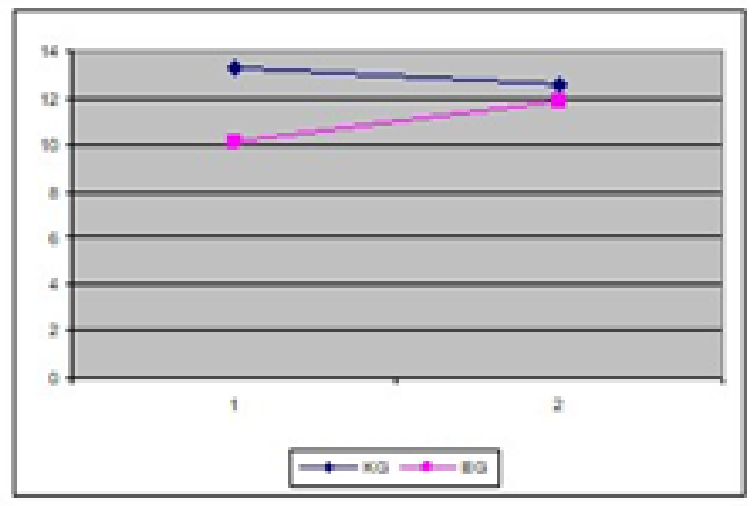

Figure 6. Dynamometry unsuitable hand

On the test „Plank”, the Control group has shown average values of incoming tests 82,3 sec, and of the out-coming ones - 79,6 sec. Decreasing of the average values by 2,07 sec has been observed. The Experimental group has shown average values of incoming tests of $41,94 \mathrm{sec}$, and of the out-coming - 57,4 sec. Improvement of the average values by 15,46 sec has been observed. After the application of the specialized fitness programme, the increasing of the average values of the „Plank” test of the Experimental group compared to the Control group is $17,53 \mathrm{~kg}$. Graphically, the results are shown on (Figure 7).

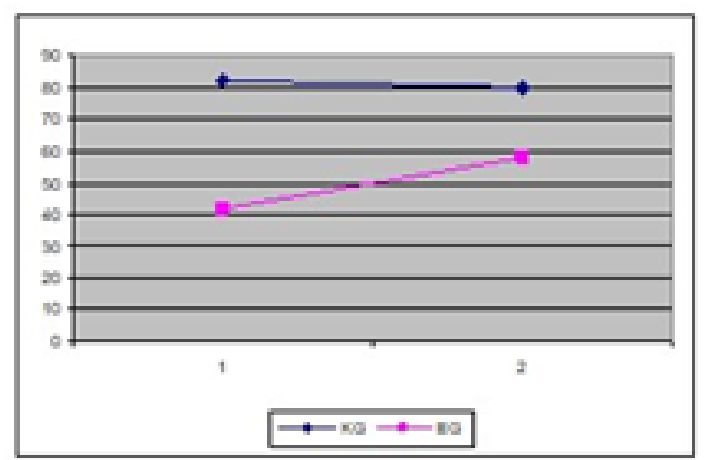

Figure 7. Plank

On the test "Shuttle 10x10”, the Control group has shown average values of incoming tests $32,42 \mathrm{sec}$, and of the out-coming ones - 33,8 sec. Increasing of the average values by 1,38 sec has been observed. The experimental group has shown average values of incoming tests of 35,62 sec, and of the out-coming - 57,4 sec. Decreasing of the average values by 1,15 sec has been observed. After the application of the specialized fitness programme, the decreasing of the average values of the „Shuttle 10x10" test of the Experimental group compared to the Control group is 2,53 sec. Graphically, the results are shown on (Figure 8).

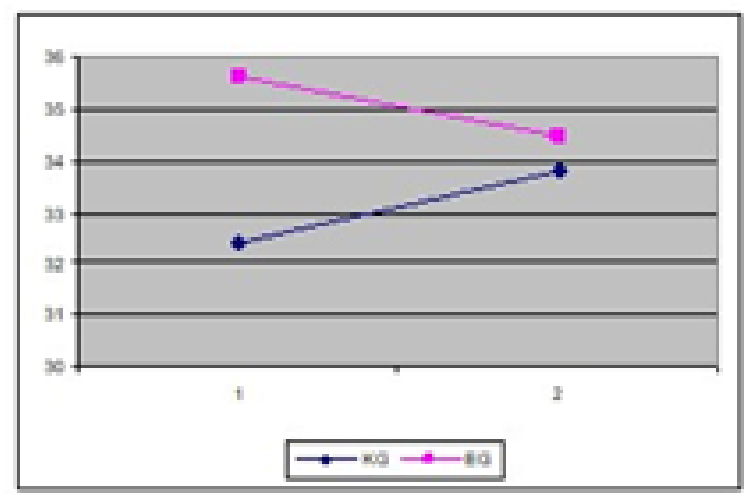

Figure 8. Shuttle 10x10

On the test „Vertical jump”, at the first measurement, the Control group has shown average values of $28,1 \mathrm{~cm}$, and on the second measurement of $27 \mathrm{~cm}$. There is decreasing of the average values by $1,1 \mathrm{~cm}$. The experimental group on the first testing has shown average values of incoming tests of 25,7 $\mathrm{cm}$, and on the second measurement $-28,4 \mathrm{~cm}$. Increasing of the results by $2,7 \mathrm{~cm}$ has been observed. After the application of the 
specialized fitness programme, the increasing of the average values of the "Vertical jump" test of the Experimental group compared to the Control group is 3,8. Graphically, the results are shown on (Figure 9).

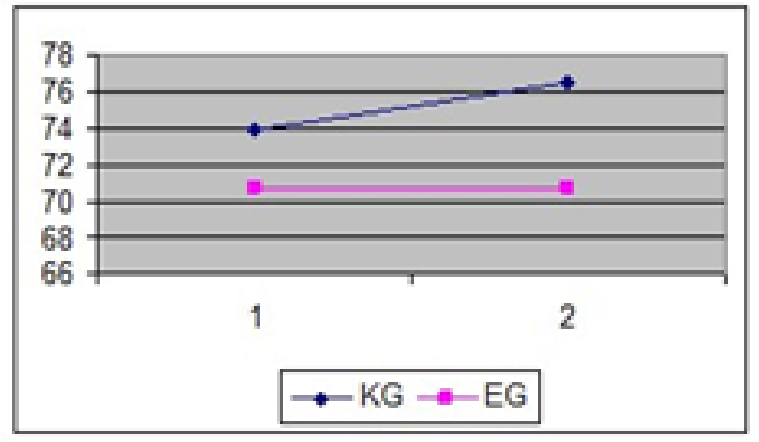

Figure 9. Vertical jump

\section{CONCLUSIONS}

1. Efficiency of the specialized fitness programme with Swiss balls for development of the physical qualities force and endurance is experimentally grounded.

2. Due to the positive influence of the oriented trainings on the new fitness programme, the results of female students from the Experimental group have increased, while the same with the Control group have been found at deadlock and even at regress.

3. The improvement of the achievements on the anthropometry and physical qualities tests, the insignificant variety with their analysis and significant increasing of the maximum results represent positively the work of lecturers and students.

\section{RECOMMENDATIONS}

Introducing of the new methods of fitness programme by using the Swiss balls is justified in view of the results achieved. At the same time, the enthusiasm and expectations out of the new and non-traditional methods increase the interest towards the knowledge on fitness.

\section{REFERENCES}

1. Rachev, K. and col. Theory and methods of Physical culture. София, NSA PRESS, 2004

2. Peeva, D., Contemporary trends in education on Physical culture and sport European perspective. Personality, Motivation, Sport; 978-954-718-521-0, 2018.

3. Peeva, D., Application of training testing in Theory and methods of education of
Physical culture. Scientific papers - Russe University "Angel Kanchev". ISSN 1311332, 2017.

4. Dimitrova, N., ,System structural analysis of the explosive force". Scientific conference "Challenges and perspectives before the sport science“, „Specifics of preparation in various sport disciplines"2017. NSA PRESS; p.190-196; Sofia, ISBN 978-954-718-492-3, 2017.

5. Peeva, D., Dynamics of physical quality "endurance" of students after Rugby trainings, from VTU "T. Kableshkov". NSA, TMFB, 2006.

6. https://www.bbteam.org/articles/3856_fitball - 24.10.2010

7. Petkov, P., Tsvetanov, T., Influence of circuit trainings of female students' endurance. Sofia, ISBN 978-954-718-4923, p. 219-228, 2017.

8. Petkov, P., Specialized fitness preparation with students. Dissertation. Veliko Tarnovo, University Sv.Sv. Cyril and Methodius, 2018.

9. Lazarov, I., Anthropometric models of Middle-distance running. Dissertation, Sofia, NSA, 2014.

10.Peltekova, I., Perkov, P., Sport and Science, Sofia, „Establishing of the level of physical development of female students from educational groups on Physical culture and sport in Trakia University ", ISSN 13103393, p. 161-169, 2/2017.

11.Petkov, P., ,Influence of circuit trainings of body mass content of female students ", Sofia, ISBN 978-954-718-492-3, p. 211219, 2017.

12.Angelova, Petya ,Therapeutic stretching with muscle-skeletal disfunction and lumbar pain. Publ. Kota, ISBN 978-954305-482-4, 2019.

13.Petya Angelova, Katya Mollova, Ivelina Angelova, STUDENT VIEW ON THE SIGNIFICANCE OF THE STRETCHING AND THE PRACTICAL APPLICATION. Academic magazine "Management and education" International Conference EDUCATION, SCIENCE, ECONOMICS AND TECHNOLOGIES 28 th - 29 th June, 2018 BURGAS.

14.Petkov, P., Angelova, P., Investigation of physiometric indices with students. Sessions schedule\&Abstracts, Ruse, Razgrad, Silistra, FRI-2G.104-1-HC-06, p. $270-271,2018$ 